\title{
A valiação da utilização de microemulsões a base de óleo de Moringa Oleífera Lam saponificado como fase contínua de fluidos de perfuração: Análise das propriedades reológicas
}

\author{
Evaluation of the use of microemulsions based on Moringa Oleifera Lam oil saponified as \\ continuous phase of drilling fluids: Analysis of rheological properties \\ Evaluación del uso de microemulsiones a base de aceite saponificado de Moringa Oleifera Lam \\ como fase continua de fluidos de perforación: Análisis de propiedades reológicas
}

Recebido: 23/03/2021 | Revisado: 29/03/2021 | Aceito: 01/04/2021 | Publicado: 12/04/2021

Gabriel Passos da Cruz

ORCID: https://orcid.org/0000-0002-9753-1398 Universidade Federal de Sergipe, Brasil E-mail: gabrielpassoscr7@hotmail.com

Walisson dos Anjos Andrade

ORCID: https://orcid.org/0000-0003-4389-3374 Universidade Federal de Sergipe, Brasil

E-mail: walissondosa.andrade@gmail.com

Larissa Alves da Silva

ORCID: https://orcid.org/0000-0002-2633-0089 Universidade Federal de Sergipe, Brasil

E-mail: larissaalves.d.s@outlook.com

Acto de Lima Cunha

ORCID: https://orcid.org//0000-0002-1923-649X Universidade Federal de Sergipe, Brasil E-mail: actolimacunha@yahoo.com.br

Gabriel Francisco da Silva

ORCID: https://orcid.org/ 0000-0002-9622-2518 Universidade Federal de Sergipe, Brasil E-mail: gabriel@ufs.br

João Paulo Lobo dos Santos

ORCID: https://orcid.org/0000-0003-2947-718X Universidade Federal de Sergipe, Brasil E-mail: jplobo2011@gmail.com

\begin{abstract}
Resumo
Na exploração petrolífera, uma das principais atividades é a perfuração de poços, na qual faz-se necessário o uso dos chamados fluidos de perfuração. Devido a sua grande importância, tem-se buscado desenvolver fluidos de perfuração com propriedades melhoradas e, nesse intuito, estudos mostram que sistemas microemulsionados podem ser viáveis para esta aplicação, visto que os mesmos são mais estáveis que os sistemas emulsionados, o que os tornam mais eficientes. O objetivo deste trabalho foi desenvolver um fluido de perfuração utilizando como sua fase continua sistemas microemulsionados à base de óleo de Moringa Oleífera Lam saponificado. No desenvolvimento do sistema microemulsionado foi utilizado água destilada (fase aquosa), óleo de moringa (fase oleosa), óleo de moringa saponificado (tensoativo) e n-butanol (cotensoativo) e uma razão Cotensoativo/Tensoativo igual a 1. Após o preparo do sistema microemulsionado foram adicionados os aditivos: Goma Xantana (viscosificante) e Baritina (adensante). Para a caracterização do fluido foram realizados estudos reológicos para determinar parâmetros como o limite de escoamento, o índice de consistência, o índice de comportamento e o grau de tixotropia, através da variação da concentração do viscosificante em $4 \%, 6 \%$ e $8 \%$. Os resultados obtidos mostraram que os fluidos apresentaram comportamento pseudoplástico, seguindo o modelo de Herschell-Bulkley e de acordo com outros estudos envolvendo fluidos microemulsionados. Além disso, com o aumento do percentual de viscosificante, foram observadas melhorias nas propriedades do fluido, como limite de fluxo e grau tixotrópico.
\end{abstract}

Palavras-chave: Óleo de Moringa Oleífera Lam; Microemulsão; Fluido de perfuração; Parâmetros reológicos.

\footnotetext{
Abstract

In oil exploration, one of the main activities is the drilling of wells, in which it is necessary to use the so-called drilling fluids. Due to its great importance, it has been sought to develop drilling fluids with improved properties and,
} 
to that end, studies show that microemulsified systems can be viable for this application, since they are more stable than emulsified systems, which makes them more efficient. The objective of this work was the development of a drilling fluid using as its continuous phase microemulsioned systems based on Moringa Oleifera Lam saponified oil. In the development of the microemulsioned system were used distilled water (aqueous phase), moringa oil (oily phase), saponified moringa oil (surfactant) and n-butanol (cotensoative) and a Cotensoative/Surfactant ratio equal to 1 were used. After the preparation of the microemulsioned system, additives were used to prepare drilling fluid: Xanthan Gum (viscosifier) and Baritin (thickening agent). In order the characterization of the fluid, rheological studies were carried out to determine parameters such as the flow limit, behavior index, consistency index and the degree of thixotropy, by varying the viscosifier concentration by $4 \%, 6 \%$ and $8 \%$. The results obtained showed that the fluids showed pseudoplastic behavior, following the Herschell-Bulkley model and according to other studies involving microemulsioned fluids. In addition, with the increase in the percentage of viscosifier, improvements were observed in the properties of the fluid, such as flow limit and thixotropic degree.

Keywords: Moringa Oleifera Lam oil; Microemulsion; Drilling fluid; Rheological parameters.

\section{Resumen}

En la exploración petrolera, una de las principales actividades es la perforación de pozos, en la que es necesario utilizar los llamados fluidos de perforación. Debido a su gran importancia, se ha buscado desarrollar fluidos de perforación con propiedades mejoradas y, para ello, estudios demuestran que los sistemas microemulsificados pueden ser viables para esta aplicación, ya que son más estables que los sistemas emulsionados, lo que los hace más eficientes. El objetivo de este trabajo fue el desarrollo de un fluido de perforación utilizando sistemas microemulsionados a base de aceite saponificado de Moringa Oleifera Lam como su fase continua. En el desarrollo del sistema microemulsionado se utilizó agua destilada (fase acuosa), aceite de moringa (fase aceitosa), aceite de moringa saponificado (tensioactivo) y n-butanol (agente coactivo) y una relación Cotensoativo / Tensoactivo igual a 1. Después de la preparación del En el sistema microemulsionado, se agregaron aditivos: Goma Xantana (viscosificante) y Baritina (espesante). Para la caracterización del fluido se realizaron estudios reológicos para determinar parámetros como el límite de flujo, índice de comportamiento, índice de consistencia y grado de tixotropía, variando la concentración de viscosificante en $4 \%, 6 \%$ y $8 \%$. Los resultados obtenidos mostraron que los fluidos mostraron un comportamiento pseudoplástico, siguiendo el modelo de Herschell-Bulkley y de acuerdo con otros estudios con fluidos microemulsificados. Además, con el aumento en el porcentaje de viscosificantes, se observaron mejoras en las propiedades del fluido, como límite de flujo y grado tixotrópico.

Palabras clave: Aceite de Moringa Oleífera Lam; Microemulsión; Fluido de perforación; Parámetros reológicos.

\section{Introdução}

$\mathrm{Na}$ exploração de petróleo, umas das principais etapas desenvolvidas é a perfuração de poços com o objetivo de produção de óleo e gás, bem como da injeção de fluidos que auxiliem na recuperação de mais hidrocarbonetos (Souza, Luporini \& Rigoli, 2017). A perfuração de poços tem conseguido, por meio dos avanços tecnológicos, alcançar os objetivos pré-determinados em regiões de alta complexidade, principalmente em regiões offshore que oferecem naturamente mais desafios a essa etapa. Para que esta atividade petrolífera alcance um alto índice de eficiência, realizando suas operações pertinentes de forma segura, econômica e ambientalmente sustentável, faz-se necessário o uso de fluidos de perfuração, comumente chamados de lamas de perfuração. De acordo com Thomas (2004) os fluidos de perfuração são misturas complexas que, do ponto de vista químico, podem assumir aspectos de suspensão, dispersão coloidal ou emulsão, dependendo do estado físico que se encontram os componentes.

Dentre as funções a serem desempenhadas pelos fluidos de perfuração estes deve fornecer estabilidade as paredes dos poços que estão sendo perfurados, exercer a função de resfriar a broca e os equipamentos da coluna de perfuração, assim como carrear os cascalhos triturados até a superfície para posterior estudo e gerenciamento (Souza, Luporini \& Rigoli, 2017; Thomas, 2004). Além disso, os fluidos de perfuração devem minimizar a quantidade de filtrado que invadem a formação, pois pode causar dano a rocha, assim como comprometem a segurança da operação (Silva et al., 2019). De maneira geral e com base no seu constituinte principal, os fluidos são classificados em base água, base óleo e base gás. Com o avanço em pesquisas, uma nova geração de fluidos de perfuração tem sido estabelecida na qual a base do fluido é constituída por moléculas orgânicas sintéticas. Para que os fluidos de perfuração possam desempenhar suas funções de forma eficiente suas propriedades 
reológicas são extremamente importantes (Gray, Darley \& Caenn, 2014), assim como permita o alcance do sucesso na perfuração do poço (Ramalho, Souza \& Ferreira, 2021).

Os fluidos sintéticos surgiram a partir da necessidade de fluidos que possuíssem características semelhantes aos fluidos base óleo, porém, que causassem menos danos ambientais, sendo menos tóxicos e mais biodegradáveis (Mairs et al., 2000). Estes podem ser utilizados em substituição as lamas base óleo mantendo suas vantagens e, possuem características de manuseio e descarte das lamas base água (Caenn \& Chillingar, 1996). Assim, tornam uma alternativa ambientalmente mais correta em relação aos sistemas tradicionais. Nesse contexto, os sistemas microemulsionados possuem alta empregabilidade na indústria, por serem termodinamicamente estáveis, de fácil preparo e ocorre de forma espontânea (Silva, 2011), e podem ser aplicados em fluidos de perfuração de base aquosa, pois são biodegradáveis e de baixo custo de produção (Garnica et al., 2020).

Microemulsões são sistemas de misturas de óleo, água, tensoativo e, às vezes, cotensoativo, que podem distinguir-se pela polaridade da fase dispersa (Garnica et al., 2020; Curbelo et al., 2021). Elas diferem-se das emulsões por possuírem tamanho estrutural muito menor, apresenta grande estabilidade termodinâmica e aspecto transparente. Devido à estabilidade conferida aos fluidos, estes conseguem durante as paradas de circulação manter por mais tempo os cascalhos em suspensão, dificultando que os mesmos sejam depositados no fundo do poço (Curbelo et al., 2016). Recentemente alguns estudos têm sido realizados com enfoque no comportamento e caracterização das propriedades reológicas de fluidos de perfuração microemulsionados preparados à base de óleo vegetal, como exemplos o óleo de pinho descrito por Borges (2014) e o óleo de soja comentado por Ratkievicius (2015). O fluido de perfuração microemulsionado desenvolvido por Curbelo et al. (2016) apresentou caracteristicas favoráveis de viscosidade e força gel, bem como baixa perda de fluido para a formação.

Um tipo de óleo que vem sendo estudado para formulação de sistemas microemulsionados é o óleo de Moringa Oleífera Lam (Andrade et al., 2020). O óleo obtido a partir das sementes da moringa apresenta alta resistência à oxidação devido a presença do ácido Oleico (Lucena, Silveira e Costa, 2016). A escolha de óleos vegetais como substitutos ao óleo diesel e à parafina tradicionalmente utilizados se justifica por possuírem toxicidade mais baixa, sendo menos poluente ao meio ambiente. De acordo com Lucena, Silveira e Costa (2016), o óleo da Moringa Oleífera Lam é oriundo de fonte renovável e biodegradável, podendo ser utilizado como "aditivo verde" em formulações. Diante desse contexto, este trabalho tem o objetivo avaliar a utilização de óleo de Moringa Oleífera Lam no desenvolvimento de sistemas microemulsionados para utilização como fase contínua do fluido de perfuração microemulsionado. Para caracterização do fluido desenvolvido a base de óleo vegetal foram determinadas suas propriedades reológicas, bem como foi avaliada a influência da concentração de goma xantana (GX) na reologia do fluido microemulsionado.

\section{Metodologia}

Para realização deste estudo foi conduzida uma pesquisa laboratorial e do tipo quantitativa de forma a verificar a influência da concentração do viscosificante nas características reológicas do fluido de perfuração formulado. As propriedades obtidas para os fluidos, assim como os modelos que melhor descrevem seu comportamento foram realizadas com base na literatura (Machado, 2002). Os principais reagentes utilizados neste trabalho foram empregados visando atender as necessidades da formulação de um fluido de perfuração, bem como suas funções conforme especificados na Tabela 1. O nButanol utilizado foi da marca Dinâmica (P. A.), o óleo de Moringa Oleífera foi obtido das sementes da planta através do processo de prensagem e os aditivos empregados foram a Goma Xantana e a Baritina. 
Tabela 1. Reagentes utilizados na obtenção do sistema microemulsionados e produção do fluido.

\begin{tabular}{l|l}
\hline \multicolumn{1}{c|}{ Aditivos } & \multicolumn{1}{c}{ Função } \\
\hline Água destilada & Fase aquosa \\
Óleo de Moringa Oleífera Lam & Fase oleosa \\
Sabão de Óleo de Moringa Saponificado (OMOS) & Tensoativo \\
n-Butanol & Cotensoativo \\
Goma Xantana & Viscosificante \\
Baritina & Adensante \\
\hline
\end{tabular}

Fonte: Autores.

A obtenção do tensoativo a base de óleo de moringa saponificado e a composição da microemulsão foram definidas com base no estudo de Andrade et al. (2020), assim como o preparo do sistema microemulsionado seguiu as metodologias disponíveis na literatura (Souza et al. 2015; Souza et al., 2016).

\subsection{Seleção do diagrama de fases}

Estudos anteriores mostraram que à medida que ocorre a diminuição da razão $\mathrm{C} / \mathrm{T}$, a região de microemulsão torna-se mais evidente (Andrade et al., 2020). Diante disso, apesar de o tensoativo ser uma das partes mais onerosas do sistema microemulsionado, optou-se por escolher para estudo o diagrama com $\mathrm{C} / \mathrm{T}=1$ devido a sua maior região de WIV, concedendo maior confiabilidade na obtenção dos sistemas microemulsionados. A escolha do ponto dentro da região de microemulsão foi baseada no percentual mássico de seus componentes, buscando-se um ponto em que houvesse grande presença da fase aquosa, comumente observado nos fluidos base água e, também, para que fosse facilitado a dissolução do tensoativo OMOS, pois, o mesmo se encontra no estado sólido em temperatura ambiente $\left(25^{\circ} \mathrm{C}\right)$. A Figura 1 mostra o diagrama pseudotérnário, o ponto escolhido e a concentração de cada componente para a formulação do sistema microemulsionado que foi utilizado como fase contínua do fluido de perfuração a ser desenvolvido.

Figura 1. Ponto escolhido na região Winsor IV para elaboração do fluido de perfuração.

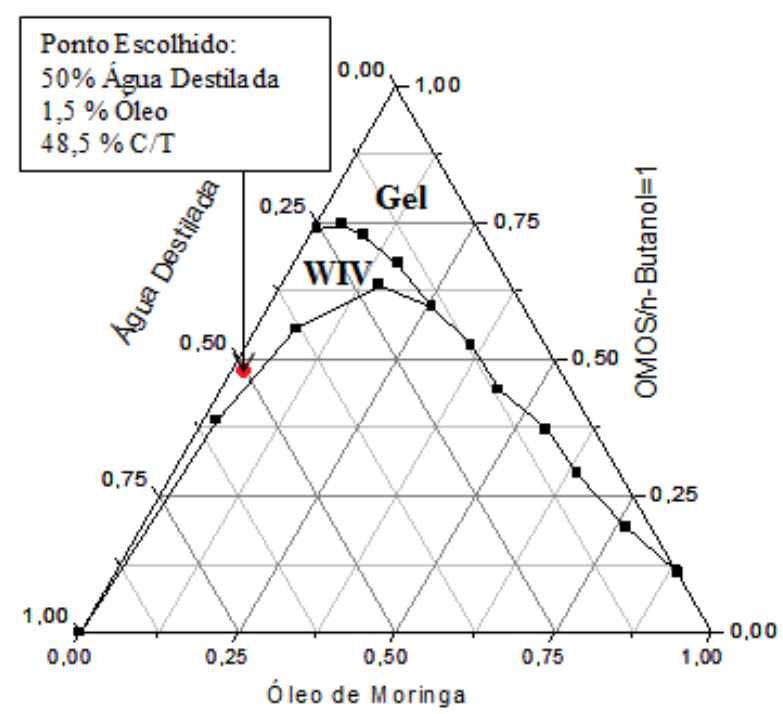

Fonte: Modificado de Andrade et al. (2020). 
Como pode ser observado da composição do sistema microemulsionado definido a partir da Figura 1 a fase contínua do fluido será rica em água, no entanto, a mesma será em forma de microemulsão o que poderá conferir uma maior estabilidade térmica comparado com outros fluidos de perfuração contendo apenas água na fase contínua. Além disso, a fração da fase oleosa será pequena (apenas $1,5 \%$ ) e o restante do óleo de moringa será utilizado na forma saponificada como tensoativo.

\subsection{Preparo do fluido de perfuração}

Foi escolhido o ponto dentro da região de microemulsão (Winsor IV), conforme apresentado na Figura 1, com as percentagens de seus constituintes, para serem feitas análises e preparar os fluidos de perfuração. Foi adotado como base de cálculo o valor de $200 \mathrm{~g}$ para preparo dos fluidos com a composição desejada, conforme o procedimento proposto por Ratkievicius (2015), como segue: a) adicionou-se, sob agitação, o volume da fase aquosa; adicionou-se lentamente a goma xantana (viscosificante), sob agitação, deixando em seguida o fluido em repouso por 24 horas para hidratação do viscosificante; b) adicionou-se a fase óleo e o emulsificante (tensoativo) à mistura anterior e, em seguida, deixa-se o fluido em agitação por 15 minutos e, posteriormente, transfere-se o fluido obtido para uma proveta, pesou-se a massa do fluido no volume determinado pela proveta e calculou-se a densidade.

Para calcular a quantidade de baritina (adensante) necessária para elevar a densidade do fluido ao valor desejado de 9 libras/galão, utilizado neste trabalho, realizou-se um balanço de massa (Equação 1). Segundo a norma da Petrobras N-2604 (Petrobras, 1998) a massa específica estabelecida para fluidos de perfuração deve estar no range de 1 a $2 \mathrm{~g} / \mathrm{cm}^{3}$ que corresponde ao range de 8,34 a 16,68 $\mathrm{lb} / \mathrm{gal}$; e para atingir esses valores acrescenta-se a baritina ao fluido no misturador e deixa-se agitar por 15 minutos.

$$
\rho \mathrm{V}+\rho_{\text {Baritina }} \mathrm{V}_{\text {Baritina }}=\rho_{\mathrm{D}} \mathrm{V}_{\mathrm{D}}
$$

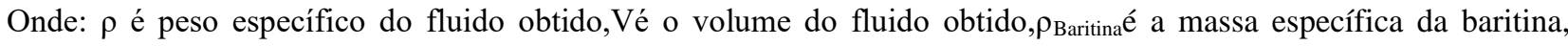

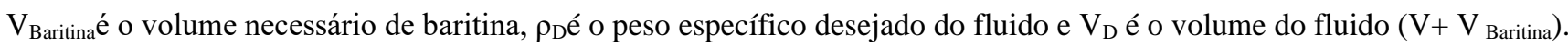

A massa da baritina ( $m_{\text {Baritina) }}$ a ser adicionada ao sistema foi obtida através do conhecimento do volume da baritina obtido pelo balanço de massa, e calculada a partir da Equação (2):

$$
\mathrm{m}_{\text {Baritina }}=\rho_{\text {Baritina }} \mathrm{V}_{\text {Baritina }}
$$

Após o cálculo, a massa de baritina calculada foi adicionada ao fluido no misturador, continuando com a agitação para total mistura do sistema.

A influência do viscosificante na reologia do fluido de perfuração microemulsionado foi investigada variando a contração de goma xantana na composição em $6 \%, 7 \%$ e $8 \%$. Após a finalização do preparo de cada fluido foram avaliadas suas propriedades reológicas.

\subsection{Ensaios reológicos}

Os ensaios para determinar os parâmetros reológicos e gelificantes dos fluidos obtidos, tais como viscosidade plástica, limite de escoamento e tixotropia, foram realizados utilizandoo Reômetro da Lamy Technology modelo RM300 acoplado ao banho termostatizado da Tecnal. Para as análises optou-se pela utilização do bulbo BOB DIN 125 (cilindro interno) e um rotor (cilindro externo), cujo range de viscosidade que o mesmo permite determinar compreende os valores entre 0,020 e 70 Pa.s e, em cada análise, foi utilizado $20 \mathrm{ml}$ de amostra dentro do copo adequado para ser utilizado junto com o BOB escolhido em 
uma temperatura de $25^{\circ} \mathrm{C}$.

As curvas de fluxo para a determinação da viscosidade plástica e limite de escoamento foram obtidas através do método CR (taxa de cisalhamento controlada), utilizando um sistema de medidas com cilindro coaxial, o qual possui um diâmetro de medida igual a $25 \mathrm{~mm}$, no intervalo de taxa de deformação de 5 a $1020 \mathrm{~s}^{-1}$, durante um minuto.

Para caracterização tixotrópica, a metodologia escolhida foi a chamada histerese, conforme Green e Weltmann (1946). Nesse experimento, primeiramente o fluido é submetido a um aumento da taxa de cisalhamento até um valor máximo e, em seguida, a uma redução da taxa de cisalhamento até zero. O resultado inicialmente esperado para um fluido newtoniano é que o caminho de ida e o de volta sejam idênticos. Porém, para um fluido tixotrópico isso não acontece. A área entre as curvas de ida e volta determina a magnitude da tixotropia. Se as curvas forem coincidentes, o efeito de tixotropia é nulo (Santos, 2013).

Na determinação do grau de tixotropia, foram necessários três minutos de experimento, no primeiro minuto variou-se a taxa de cisalhamento de 5 a $1020 \mathrm{~s}^{-1}$ (curva de ida), no segundo minuto manteve-se a taxa de cisalhamento em $1020 \mathrm{~s}^{-1}$, e no terceiro minuto variou-se a taxa de cisalhamento de 1020 a $5 \mathrm{~s}^{-1}$ (curva de volta). Por fim, foi calculada a área do ciclo de histerese, caracterizando o grau de tixotropia do fluido.

Com o intuito de avaliar o comportamento dos fluidos obtidos quando sujeitos a diferentes temperaturas, foi realizado um estudo térmico nas seguintes temperaturas $25,30,35,40,45$ e $50{ }^{\circ} \mathrm{C}$, plotando-se uma curva de viscosidade para cada temperatura utilizada.

Após a realização dos ensaios foram utilizados modelos reológicos para caracterização dos fluidos microemulsionados. Um dos modelos reológicos mais simples e muito utilizado que melhor caracteriza fluidos de perfuração é o modelo reológico de Herschel-Bulkley, que se trata do modelo de potência modificado. Este é um modelo que caracteriza o fluxo de fluidos não newtonianos utilizando três parâmetros reológicos, a saber: a tensão limite de escoamento ( $\left.\tau_{0}\right)$, o índice de consistência $(k)$ e o índice de fluxo (n), conforme mostrado na Equação (3) (Machado, 2002; Souza, Luporini e Rigoli, 2017):

$$
\tau=\tau_{0}+k \gamma^{n}
$$

Onde: $\tau$ é a tensão cisalhante e $\gamma$ é a taxa de cisalhamento.

Devido ao fato de trabalhar com três parâmetros os dados experimentais, geralmente, se ajusta melhor a este modelo, sendo por isso um dos modelos reológicos mais utilizados na indústria do petróleo para caracterizar o escoamento dos fluidos de perfuração (Machado, 2002).

Outro modelo disponível na literatura e que foi utilizado neste trabalho a título de comparativo é o de Modelo de Ostwald de Waale ou de Potência. A tensão de cisalhamento em um fluido de potência está relacionada à taxa de cisalhamento através da Equação (4) (Machado, 2002):

$$
\tau=k \gamma^{n}
$$

A viscosidade aparente foi determinada para a máxima taxa de cisalhamento utilizida nos ensaios do reômetro e a viscosidade plástica foi obtida a partir do cálculo da deformação em duas tensões de cisalhamento conhecidas. A regressão dos dados para caracterização do fluido desenvolvido em relação aos modelos reológicos foi realizada utilizando o software SciDavis.

\section{Resultados e Discussão}

As análises reológicas do ponto escolhido para elaboração do fluido de perfuração foram divididas em duas partes: influência da concentração do viscosificante sobre o fluido de perfuração e estudo térmico na amostra com melhor desempenho. 


\subsection{Influência da concentração de viscosificante}

Foram realizados estudos reológicos dos fluidos obtidos com diferentes percentuais de goma xantana com o objetivo de comparar e determinar qual percentual que mais se adequava para uso na formulação do fluido de perfuração. Os ensaios foram realizados com $4 \%, 6 \%$ e $8 \%$ do viscosificante goma xantana em temperatura ambiente $\left(25^{\circ} \mathrm{C}\right)$. Nas Figuras 2 e 3 podese observar o comparativo entre as curvas de fluxo e viscosidade aparente em função da taxa de cisalhamento com a mudança na concentração do viscosificante.

Figura 2. Curvas da taxa de cisalhamento para diferentes concentrações de Goma Xantana.

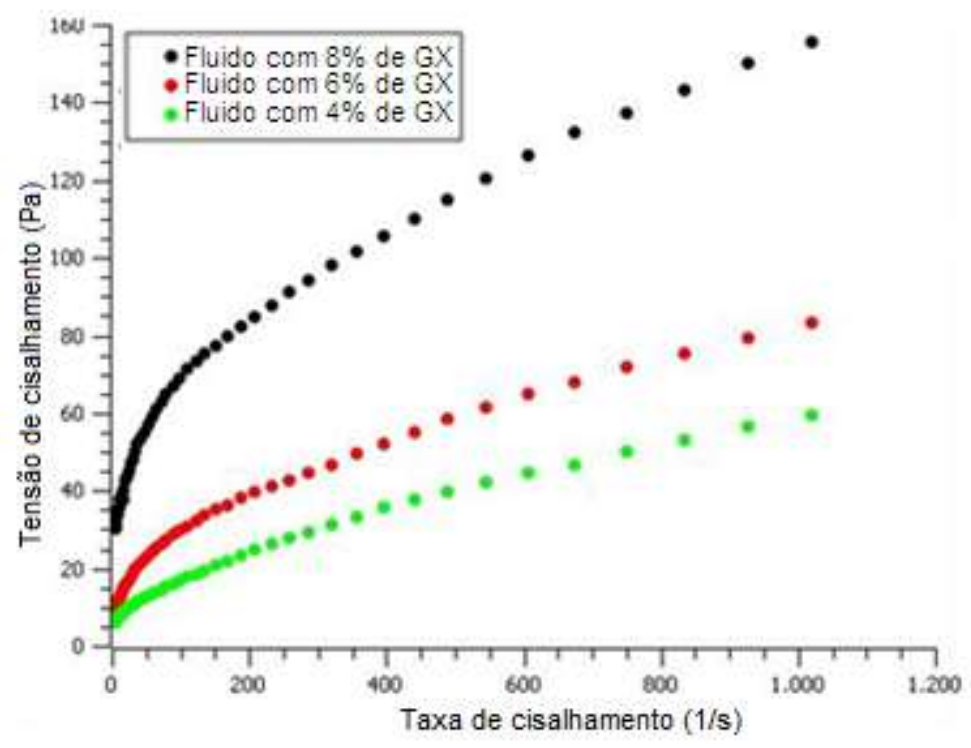

Fonte: Autores.

A partir da Figura 2 pode-se observar que os fluidos apresentaram comportamento não-Newtoniano, uma vez que, a relação de tensão cisalhante por taxa de cisalhamento não é constante, ou seja, não obedece um modelo linear. O aumento percentual de goma xantana no fluido de perfuração proporciona um acréscimo no valor do limite de escoamento inicial. Fluidos de perfuração com elevado limite de escoamento inicial possuem aplicação interessante em poços petrolíferos que possuem regiões alargadas, pois nessas regiões ocorre redução da velocidade de escoamento, devido ao aumento do diâmetro do poço, favorecendo a decantação de sedimentos e, assim, o fluido com elevado $\tau_{0}$ desenvolvido neste trabalho adquire o comportamento de fluxo tampão. Observa-se no escoamento tampão que existe deslocamento relativo próximo às paredes do poço, mas a região central do fluido move-se como um corpo sólido, sem apresentar deslocamento relativo no centro. O fluxo tampão só ocorre para fluidos não-Newtonianos, que necessitem uma tensão mínima (limite de escoamento) para causar o deslocamento relativo entre as camadas, ou seja, o fluido é altamente resistente ao escoamento (Machado, 2002).

$\mathrm{Na}$ Figura 3 é possível observar que a viscosidade aparente dos fluidos aumenta quando ocorre um acréscimo percentual de viscosificante no meio e diminui quando a taxa de cisalhamento é elevada gradativamente, ficando praticamente constante. Este comportamento caracteriza o polímero como um modificador reológico com características pseudoplásticas, o que mostra que a Goma Xantana possui fortes interações intramoleculares, o que faz deste polímero um aditivo bastante utilizado na formulação de fluidos de perfuração (Melo, 2008). Segundo Diaz, Vendruscolo e Vendruscolo (2004), algumas pesquisas mostraram que polímeros ramificados (como a goma xantana) possuem alta capacidade viscosificante, devido ao aumento das ramificações que são longas e, enrolam-se entre as cadeias. 
Figura 3. Curvas de viscosidade aparente para diferentes concentrações de Goma Xantana.

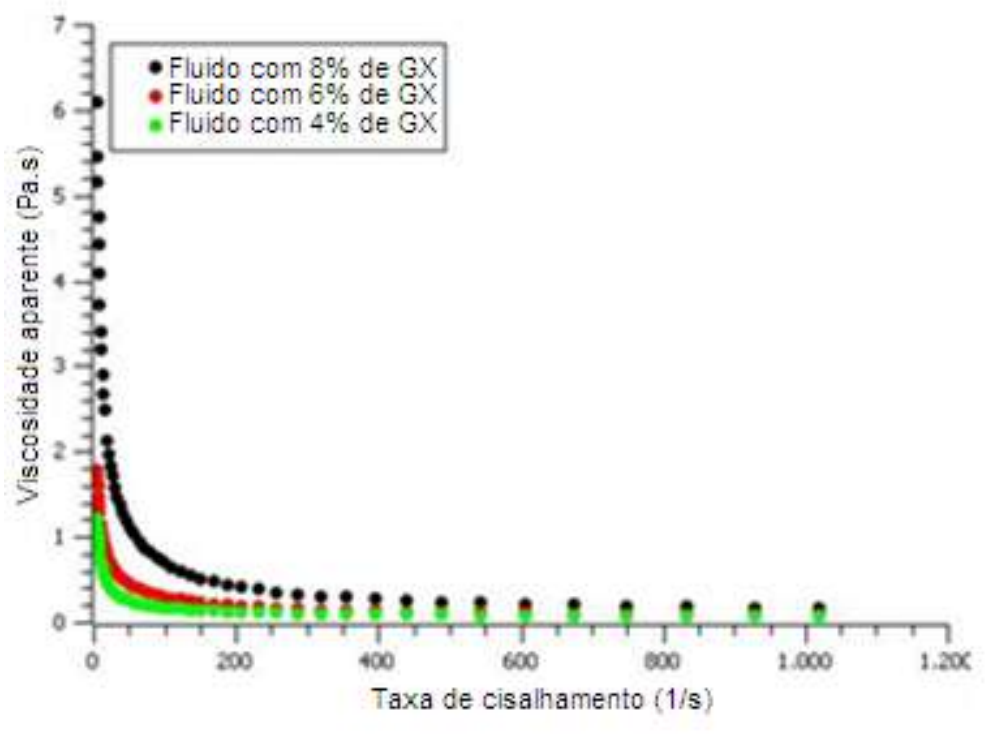

Fonte: Autores.

O aumento da viscosidade aparente do fluido de perfuração durante sua utilização tem como consequência a redução da velocidade de sedimentação das partículas, porém, não se pode aumentar demasiadamente a viscosidade, pois, ocasionará em problemas como: o aumento do teor de sólidos, enceramento da broca de perfuração, redução da taxa de penetração e aumento da perda de carga. Já a redução da viscosidade com o incremento da taxa de cisalhamento deve-se ao fato das partículas dispersas nos sistemas pseudoplásticos, em repouso, estarem de forma irregular, proporcionando ao fluido uma alta resistência ao fluxo caracterizada pelos altos valores de viscosidade. Segundo Melo (2008) ao aumentar a taxa de cisalhamento essas partículas se orientam linearmente na direção do fluxo, diminuindo assim sua viscosidade. Sousa et al. (2020) afirmam que a redução da viscosidade aparente com ao incrementar a taxa de cisalhamento é um comportamento desejável, visto que em altas taxas de cisalhamento, que ocorrem durante a perfuração, irá reduzir as pressões de bombeio, assim como poderá favorecer a capacidade de transporte dos cascalhos pelo fluido de perfuração até a superfície, o que melhora a limpeza do poço.

Na Tabela 2 estão organizados os valores dos parâmetros calculados pelos modelos reológicos de Herschell-Bulkley e Ostwald de Waale, além de seus coeficientes de correlação, para o fluido formulado com Goma Xantana como viscosificante.

Tabela 2. Valores dos parâmetros e coeficiente de relação para os modelos de Herschell-Bulkley e Ostwalde de Walle.

\begin{tabular}{|c|c|c|c|c|c|c|c|}
\hline \multirow{3}{*}{$\begin{array}{l}\text { Concentração de } \\
\text { GX }(\%)\end{array}$} & \multirow{2}{*}{\multicolumn{3}{|c|}{$\begin{array}{l}\text { Herschell-Bulkley } \\
\tau=K \gamma^{n}+\tau_{0}\end{array}$}} & \multirow[t]{3}{*}{$\mathbf{R}^{2}$} & \multirow{2}{*}{\multicolumn{2}{|c|}{$\begin{array}{l}\begin{array}{l}\text { Ostwalde de } \\
\text { Walle }\end{array} \\
\boldsymbol{\tau}=\boldsymbol{K} \boldsymbol{\gamma}^{n} \\
\text { Parâmetros }\end{array}$}} & \multirow[t]{3}{*}{$\mathbf{R}^{2}$} \\
\hline & & & & & & & \\
\hline & $K$ & $\eta$ & $\tau_{0}$ & & $K$ & $\eta$ & \\
\hline 4 & 0,79 & 0,610 & 2,40 & 0,999 & 2,64 & 0,427 & 0,981 \\
\hline 6 & 3,44 & 0,450 & 4,20 & 0,999 & 4,72 & 0,404 & 0,998 \\
\hline 8 & 9,20 & 0,391 & 13,0 & 0,998 & 17,4 & 0,304 & 0,994 \\
\hline
\end{tabular}

Fonte: Autores.

É possível observar através dos coeficientes de correlação $\left(\mathrm{R}^{2}\right)$ obtidos, que os modelos utilizados na análise reológica 
dos fluidos preparados se ajustaram bem aos resultados experimentais, possuindo valores próximos da unidade. A Tabela 2 mostra ainda que o modelo de Herschell-Bulkley foi o que mais se adequou para as concentrações utilizadas e está coerente com estudos envolvendo fluidos microemulsionado como pode ser visto na literatura (Curbelo et al., 2016; Sousa et al., 2020) e até nanofluido (Souza et al., 2020). Os resultados também estão de acordo com outros trabalhos encontrados na literatura onde foi observado um aumento do limite de escoamento em fluidos de perfuração utilizando suspensões argilosas salinas e goma xantana como viscosificante (Souza, Luporini e Rigoli, 2017).

Infere-se dos valores do índice de comportamento $(\eta)$, que os fluidos obtidos possuem características pseudoplásticas, uma vez que, os seus valores estão compreendidos na faixa de $0<\eta<1$, quando submetidos em temperatura ambiente $\left(25^{\circ} \mathrm{C}\right)$. Baixos valores de $\mathrm{n}$ são desejáveis em fluidos de perfuração para facilitar a injeção do fluido e melhorar a taxa de perfuração (Candia e Deckwer, 1999). O valor do índice de comportamento indica o grau de pseudoplasticidade do fluido e, assim, indica o quanto o fluido "afina" com o incremento da taxa de cisalhamento. Quanto menor o valor de $\eta$, menor é o valor da viscosidade aparente com o incremento da taxa de cisalhamento e, portanto, menor resistência o fluido apresenta para escoar (Machado, 2002).

Relacionado às concentrações de Goma Xantana utilizadas, foram realizados ensaios tixotrópicos. Através do software utilizado, foram obtidas curvas de fluxo ao se aumentar uniformemente a taxa de cisalhamento e, depois, reduzi-la uniformemente para avaliar se apresentam duas curvas distintas (histerese). Esta caracterização é observada através de redução significativa na viscosidade sob taxa de cisalhamento constante em função do tempo, e pela recuperação da viscosidade pelo fluido quando ocorre a suspensão do cisalhamento (Melo, 2008).

Um bom comportamento tixotrópico dos fluidos é desejado para evitar a sedimentação dos sólidos perfurados pela broca durante sua parada para manobrar. Entretanto, se a viscosidade aumentar continuamente durante o tempo em que ocorrem paradas na perfuração, picos de pressão podem ser gerados quando a circulação recomeçar e, a pressão de fratura poderá ser alcançada. Desta forma, os fluidos de perfuração requerem uma gelificação rápida e não progressiva (Waldmann, Branco e Martins, 2007).

Dentre as curvas obtidas, a do fluido utilizando $8 \%$ em concentração de Goma Xantana, foi a que obteve melhores resultados, observando-se o maior grau tixotrópico. A Figura 4 mostra a curva de histerese para o fluido preparado com $8 \%$ de GX e a Tabela 6 mostra os valores de tixotropia para as concentrações utilizadas. Da literatura também foi encontrado que as propriedades reológicas de fluidos microemulsionados foram melhoradas com aumento da concentração de goma xantana, o que proporcionou maior pseudoplasticidade e aumento da viscosidade aparente, do limite de escoamento e da força gel (Sousa et al., 2020). 
Figura 4. Curva de histerese para o fluido preparado com $8 \%$ de GX com base na curva de ida (superior) e volta (inferior).

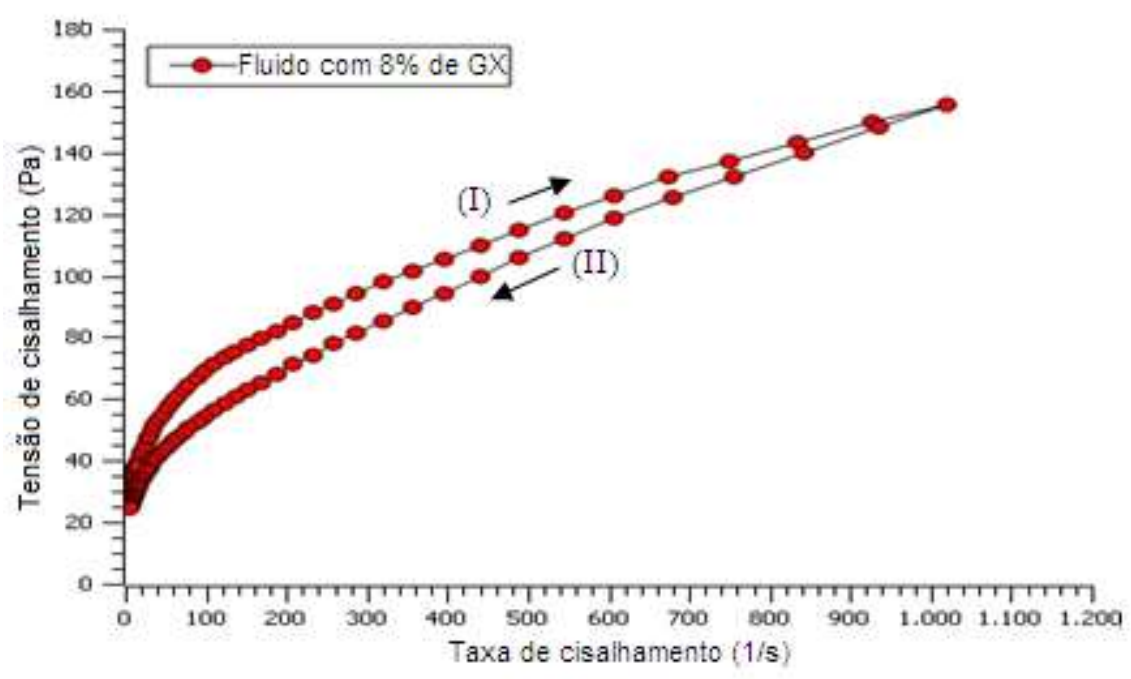

Fonte: Autores.

O software Rheomatic-P calcula o valor do grau tixotrópico por histerese, obtido pela diferença entre as rampas de ida (I), aumentando-se uniformemente a taxa de cisalhamento, e volta (II), reduzindo-se uniformemente a taxa de cisalhamento, a área formada entre as duas curvas define a magnitude da tixotropia do fluido. A Tabela 3 mostra os valores obtidos para os fluidos preparados e estas análises foram realizadas em temperatura ambiente $\left(25^{\circ} \mathrm{C}\right)$ para todas as composições.

Tabela 3. Valores do grau de tixotropia para as concentrações utilizadas.

\begin{tabular}{c|c}
\hline Concentração de GX (\%) & Tixotropia $(\mathbf{P a} / \mathbf{s})$ \\
\hline 4 & 3926 \\
\hline 6 & 6470 \\
\hline 8 & 8875 \\
\hline
\end{tabular}

Fonte: Autores.

Da Tabela 3, percebe-se que à medida que se aumenta a concentração de viscosificante, o grau de tixotropia do fluido é aumentado. Dessa forma, confirma-se as características tixotrópicas requeridas para o fluido de perfuração obtido como, por exemplo, o aumento da capacidade de sustentação dos sólidos perfurados durante as paradas de circulação, evitando assim prisão da coluna de perfuração. Este comportamento é verificado devido ao aumento da quantidade de polímero (viscosificante) no meio promover uma estruturação e alta interação das moléculas e, consequentemente, proporciona um aumento da viscosidade e grau tixotrópico da solução (Melo, 2008).

\subsection{Influência da temperatura nos parâmetros reológicos do fluido de perfuração}

O fluido selecionado para análise da influência térmica sobre as propriedades reológicas, foi aquele que possui concentração de $8 \%$ de Goma Xantana. Isso se deve ao fato observado do aumento na concentração do viscosificante ter aumentado o grau de tixotropia do fluido, o que favorece a capacidade de sustentação dos cascalhos. As Figuras 5 e 6 mostram as curvas de fluxo e viscosidade aparente em função do aumento de temperatura, respectivamente, para o fluido escolhido. 
Figura 5. Curvas de fluxo em função da temperatura para o fluido com $8 \%$ de GX.

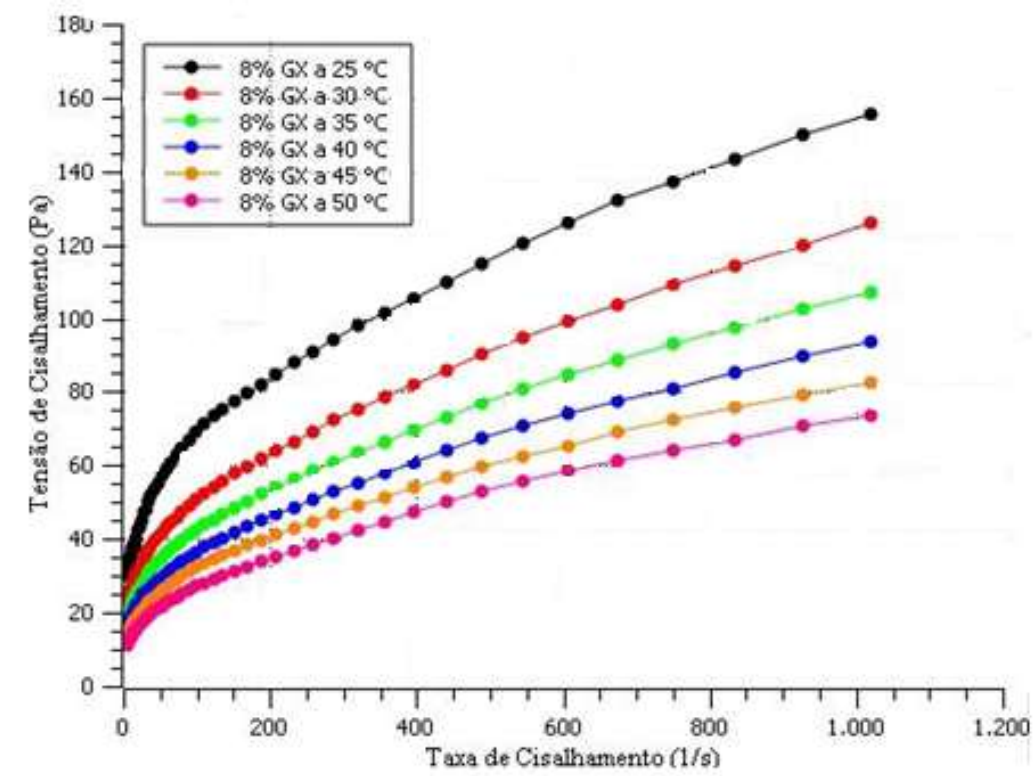

Fonte: Autores.

Através da Figura 5 e da Tabela 4, constata-se que o limite de escoamento inicial ( $\tau 0)$ do fluido diminui com o aumento da temperatura, entretanto, mantém sua viscosidade aparente em torno de um valor constante, mesmo com o aumento de temperatura e taxa de cisalhamento para valores acima de 200 s-1. Esta característica é importante, pois durante a perfuração ocorre um aumento gradual da temperatura com a profundidade devido ao gradiente geotérmico da terra, mas as características do fluido são mantidas constantes.

Figura 6. Curvas de viscosidade aparente em função da temperatura para o fluido com $8 \%$ de GX.

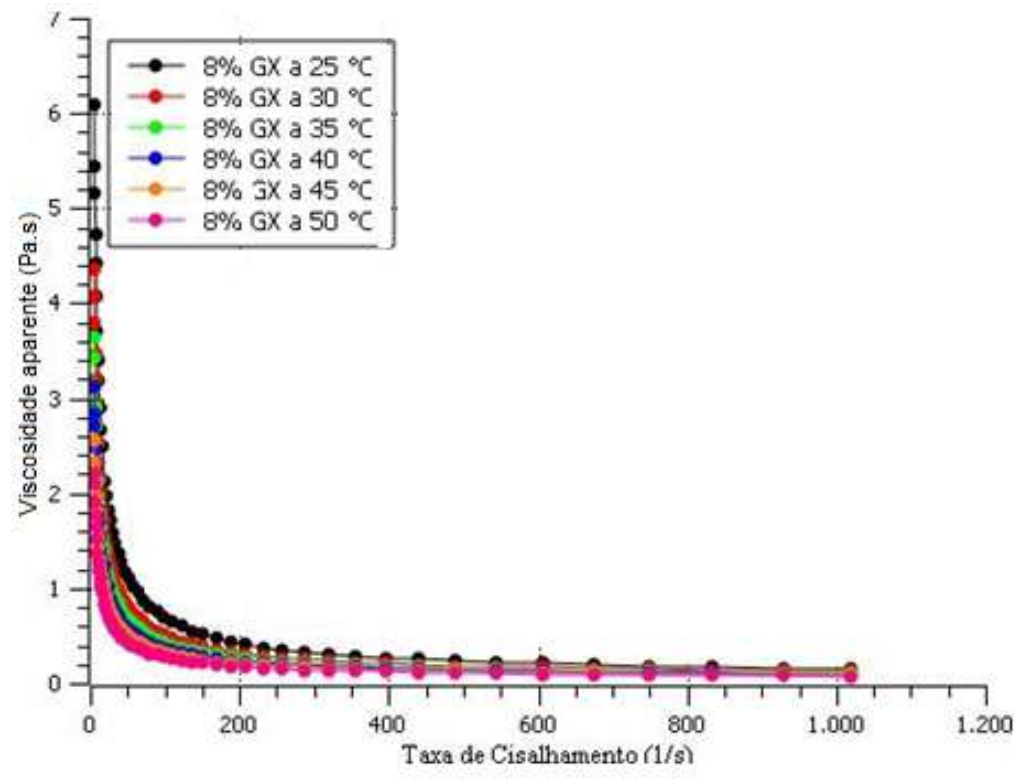

Fonte: Autores. 
Conforme verificado na seção 3.1, o modelo reológico que mais se adequou as curvas dos fluidos estudados foi o de Herschell-Bulkley. Dessa forma, foi verificado se com o aumento de temperatura o fluido com $8 \%$ de GX mantém as mesmas características reológicas através do coeficiente de correlação e, analisar os parâmetros do modelo utilizado. Os valores são mostrados a seguir na Tabela 4 .

Tabela 4. Valores dos parâmetros do modelo de Herschell-Bulkley e coeficiente de correlação em função da temperatura para o fluido com $8 \%$ de GX.

\begin{tabular}{c|c|c|c|c}
\hline \multicolumn{5}{c}{ Modelo de Herschell-Bulkley } \\
\hline T $\left({ }^{\circ} \mathbf{C}\right)$ & $\mathbf{K}$ & $\mathbf{H}$ & $\boldsymbol{\tau}_{\mathbf{0}}$ & $\mathbf{R}^{\mathbf{2}}$ \\
\hline 25 & 9,20 & 0,391 & 13,0 & 0,998 \\
\hline 30 & 5,22 & 0,438 & 11,8 & 0,999 \\
\hline 35 & 4,21 & 0,446 & 10,0 & 0,999 \\
\hline 40 & 3,51 & 0,454 & 8,60 & 0,999 \\
\hline 45 & 3,44 & 0,441 & 6,20 & 0,998 \\
\hline 50 & 2,16 & 0,492 & 6,60 & 0,999 \\
\hline
\end{tabular}

Fonte: Autores.

A partir da Tabela 4 pode-se verificar através do índice de comportamento (n), que as características pseudoplásticas do fluido com o viscosificante (Goma Xantana) são mantidas quando este é exposto a temperaturas entre 25 e $50{ }^{\circ} \mathrm{C}(0<\mathrm{n}<$ 1). Os resultados do coeficiente de correlação, $R^{2}$, mostram que o modelo utilizado representou bem as curvas obtidas experimentalmente, possuindo valores próximos da unidade. $O$ índice de consistência $(K)$ indica o grau de resistência do fluido diante do escoamento, ou seja, quanto maior o valor de $K$ mais viscoso será o fluido. Observa-se que os valores de $K$ decrescem com o aumento de temperatura, justificando que o fluido se torna menos viscoso com o incremento térmico. A alta viscosidade observada em alguns fluidos de perfuração tem utilidade particular em reservatórios à alta temperatura, onde a microemulsão continua estável, mas a viscosidade é substancialmente reduzida (Curbelo et al., 2016). Através do aquecimento, foi feita uma análise térmica para determinar o comportamento da tixotropia do fluido. Os resultados estão demonstrados na Tabela 5 .

Tabela 5. Valores de tixotropia em função do aumento de temperatura.

\begin{tabular}{c|c}
\hline Temperatura $\left({ }^{\circ} \mathbf{C}\right)$ & Tixotropia $(\mathbf{P a} / \mathbf{S})$ \\
\hline 25 & 8875 \\
\hline 30 & 3084 \\
\hline 35 & 1772 \\
\hline 40 & 1169 \\
\hline 45 & 1171 \\
\hline 50 & 1073 \\
\hline
\end{tabular}

Fonte: Autores. 
Como mostrado na Tabela 5, o grau tixotrópico do fluido diminui com o aumento da temperatura. Este resultado já era esperado, visto que, a amostra de fluido torna-se menos viscosa com a elevação térmica. De acordo com Shiroma (2012) a redução tixotrópica é influenciada pela agregação das partículas, em virtude da diminuição do número de estruturas em gel disponíveis e área superficial apta para interação entre as moléculas.

Por fim foi realizada a comparação das propriedades do fluido obtido com outros estudos utilizando fluido de perfuração microemulsionado. Para isto foram comparados os valores obtidos de viscosidade aparente (VA), viscosidade plástica (VP) e limite de escoamento (LE) encontrados na literatura de fluidos de perfuração a base de microemulsão que também utilizaram goma xantana como viscosificante, mesmas condições de temperatura e máxima taxa de cisalhamento utilizado nos estudos que foi próximo de $1020 \mathrm{~s}^{-1}$. Os valores são mostrados na Tabela 6, assim como os valores estipulados pela norma Petrobras N-2604 (Petrobras, 1998). As unidades foram convertidas, uma vez que a norma Petrobras N-2604 apresenta os valores em unidade de campo.

Tabela 6. Propriedades do fluido obtido e comparativo com a literatura.

\begin{tabular}{c|c|c|c|c}
\hline VA (cP) & VP (cP) & LE $\left(\mathrm{lbf} / 100 \mathrm{ft}^{2}\right)$ & Base & Literatura \\
\hline$>15$ & $>4$ & Máximo igual a & Água & \\
& & $15 * \mathrm{VP}$ & Microemulsão & Este trabalho \\
\hline 216,65 & 68 & 27,17 & Microemulsão & Sousa et al. (2010) \\
\hline 133,13 & 109,25 & 47,75 & Microemulsão & Gonçalves (2018) \\
\hline 40,50 & 29 & 23 & & \\
\hline
\end{tabular}

Fonte: Autores.

Da Tabela 6 é possível verificar que o fluido de perfuração obtido possui propriedades reologicas compatíveis com outros fluidos de perfuração microemulsionados encontrados na literatura. Além disso, os valores de viscosidade aparente e plástica estão de acordo com o exigido pela norma de referência para elaboração da Petrobras para elaboração de fluidos de perfuração. Assim, o fluido microemulsionado obtido a partir de óleo de Moringa Oleífera se mostra interessante para aplicação na indústria do petróleo, visto que atende as especificações desejadas, bem como pode ser formulado de um material biodegradável, o que proporciona grandes benefícios ao meio ambiente.

\section{Considerações Finais}

Nos ensaios reológicos foi realizado um estudo da concentração do viscosificante (Goma Xantana) e, verificou-se que com o aumento da concentração do polímero no meio, as propriedades reológicas do fluido foram melhoradas, como por exemplo, o aumento da viscosidade aparente. Comportamentos similares a este foram observados em trabalhos encontrados na literatura.

Os fluidos apresentaram comportamento não-Newtoniano, sendo representados pelo modelo reológico de HerschellBulkley, pois obteve o melhor ajuste, com o coeficiente de correlação (R2) próximo da unidade, caracterizando o fluido como pseudoplástico. Foi verificado que o grau de tixotropia do fluido, definido através do método de histerese, aumenta à medida que ocorre incrementos na concentração do viscosificante presente no fluido de perfuração.

Através da análise térmica, constatou-se que para o fluido com $8 \%$ de Goma Xantana o limite de escoamento inicial $(\tau 0)$ diminui com o aumento da temperatura, porém a viscosidade aparente mantém o mesmo comportamento à medida que se eleva a temperatura e a taxa de cisalhamento, já para taxa de cisalhamento inferior a aproximadamente $200 \mathrm{~s}-1$ pode-se observar uma redução na capacidade do fluido manter a viscosidade aparente com o aumento da temperatura. O 
comportamento verificado no fluido desenvolvido com aumento da temperatura é extremamente importante na perfuração de poços, visto que a viscosidade aparente do fluido de perfuração deve manter o comportamento e eficácia no transporte dos cascalhos gerados na perfuração o que aumenta a eficiência de limpeza do poço.

Fundamentado no decaimento dos valores do índice de consistência (K) do modelo de Herschell-Bulkley, observou-se que este parâmetro reforça a justificativa de que o fluido se torna menos viscoso principalmente para taxas de cisalhamento superior a $200 \mathrm{~s}-1$ com o aumento da temperatura. O grau tixotrópico dos fluidos de perfuração também diminui com a elevação térmica.

Os resultados obtidos neste trabalho mostraram que o sistema microemulsionado desenvolvido a partir de óleo de Moringa Oleífera Lam possui potencial para servir de fase contínua de fluidos de perfuração, visto que as propriedades reológicas obtidas estão em concordância com estudos encontrados na literatura, bem como com as características desejadas para fluidos de perfuração de poços estabelecida em norma. Além disso, mostra uma alternativa oriunda de uma fonte renovável e biodegradável, o que ambientalmente é melhor quando comparado com outros fluidos sintéticos utilizados pela indústria do petróleo.

Como pesquisas futuras deve ser investigado o efeito do aumento da razão cotensoativo/tensoativo sobre as propriedades do fluido de perfuração. Este aumento da razão C/T poderá acarretar em uma redução do consumo do tensoativo que é a parte mais onerosa do sistema microemulsionado, visto que se torna necessária a realização do processo de saponificação do óleo de moringa para obtenção do tensoativo. Além disso, análises térmicas do fluido desenvolvido podem ser conduzidas para verificar a sua aplicabilidade em ambientes de poço com temperaturas elevadas.

\section{Referências}

Andrade, W. A., Cruz, G. P., Santos, M. F. O., Silva, G. F., Silva, M. S. \& Santos, J. P. L. (2020). Síntese de um tensoativo a base de óleo de Moringa Oleífera Lam. e formulação de sistemas microemulsionados para a quebra de emulsões do tipo água em óleo. Research, Society and Development, 9 (2), e193922194.

Borges, T.A. (2014). Análise da Reologia e do Escoamento de um Fluido de Perfuração a partir de Microemulsão a Base de Óleo Vegetal. Trabalho de Graduação, Natal, RN.

Caenn, R. \& Chillingar, G. V. (1996). Drilling Fluids: State of the Art. Journal of Petroleum Science and Engineering, 14, $221-230$.

Candia, J.-L. F. \& Deckwer, W.-D. (1999). Effect of the Nitrogen Source on Pyruvate Content and Rheological Properties of Xanthan. Biotechnol Progress, 15: $446-452$.

Curbelo, F. D. S., Garnica, A. I. C., Nascimento, B. S. C., Leal, G. L. R., Tertuliano, T. M. \& Silva, R. R. (2021). Influence of the oleic phase and cosurfactant addition in non-ionic microemulsified systems. Research, Society and Development, v. 10, n. 2 , e58410212902.

Curbelo, F. D. S., Sousa, R. P. F., Garniça, A. I. C., Aranha, R. M., Freitas, J. C. O. \& Braga, G. S. (2016). Estudo Reológico de um Fluido de Perfuração Microemulsionado. In: XXI CONGRESSO BRASILEIRO DE ENGENHARIA QUÍMICA, Fortaleza (CE), Editora Realize.

Diaz, P. S., Vendruscolo, C. T. \& Vendruscolo, J. L. S. (2004). Reologia de Xantana: uma Revisão sobre a Influência de Eletrólitos na Viscosidade de Soluções Aquosas de Gomas Xantana. Semina: Ciências Exatas e Tecnológicas, v. 25, n. 1, 5-28.

Garnica, A. I. C., Curbelo, F. D. S., Queiroz, I. X., Araújo, E. A., Sousa, R. P. F., Paiva, E. M., Braga, G. S. \& Araújo, E. A. (2020). Desenvolvimento de microemulsões como aditivo lubrificante em fluido de perfuração. Research, Society and Development, v. 9, n. 7, e212973703.

Gonçalves, S. S. G. (2018). Desenvolvimento de fluidos de perfuração poliméricos a base de microemulsões e avaliação de suas propriedades. Dissertação de MSc, Natal, RN.

Gray, G. R., Darley, H.C.H. \& Caenn, R. (2014) Fluidos de perfuração e completação, 6 ed., Rio de Janeiro, Elsevier.

Green, H. \& Weltmann, R.N. (1946). Equations of thixotropic breakdown for rotational viscometer. Industrial \& Engineering Chemistry Analytical Edition, v. 18, n. 3, 167- 172 .

Lucena, L. C. F. L., Silveira, I. V. \& Costa, D. B. (2016). Avaliação de ligantes asfálticos modificados com óleo da Moringa Oleífera Lam para uso em misturas mornas. Revista Matéria, 11681, 72-82. 
Research, Society and Development, v. 10, n. 4, e30910414188, 2021

(CC BY 4.0) | ISSN 2525-3409 | DOI: http://dx.doi.org/10.33448/rsd-v10i4.14188

Machado, J.C.V. (2002). Reologia e escoamento de fluidos: Ênfase na indústria do petróleo, 1 ed., Rio de Janeiro, Interciência.

Mairs, H., Smith, J, Melton, R., Pasmore, F. \& Maruca, S. (2000). Efeitos Ambientais dos Cascalhos Associados a Fluidos Não Aquosos: Fundamentos Técnicos.

Melo, K. C. (2008). Avaliação e Modelagem Reológica de Fluidos de Perfuração Base Água. Dissertação de MSc, Natal, RN.

Petrobras. (1998). Viscosificante para fluido de perfuração base de água na exploração e produção de petróleo. Especificação, N-2604. Rio de Janeiro, Brasil.

Ramalho, W. J. C. R., Souza, M. F., Ferreira, H. S. (2021). Investigação do efeito viscosificante e estabilizante de argilas hidrofílica e hidrofóbica em fluidos emulsionados de base oleoso. Research, Society and Development, v. 10, n. 3, 1-13, e3910312927.

Ratkievicius, L. A. (2015). Avaliação de argila modificada por tensoativo para aplicação em fluido de perfuração a base de óleo vegetal. Dissertação de MSc, Natal, RN.

Santos, T. G. M. (2013). Avaliação de modelos de tixotropia aplicados a fluidos de perfuração. Monografia (Graduação), Curitiba, PR.

Shiroma, P. H. (2012). Estudo do comportamento reológico de suspensões aquosas de bentonita e CMC: influência da concentração do NaCl. Dissertação de MSc, São Paulo, SP.

Silva, G. C. (2011). Sistema microemulsionado: Caracterização e aplicação na indústria do petróleo. 2011. p25. Tese de DSc. Curso de Pós-Graduação em Química, Natal, RN.

Silva, S. S. S., Nóbrega, K. C., Amorim, L. V. \& Lira, H. L. (2019). Avaliação de amidos modificados empregados na mineração para aplicação em fluidos de perfuração de poços de petróleo. Revista Matéria, v.24, n.03, e-12427.

Sousa, R. P. F., Curbelo, F. D. S., Garnica, A. I. C., Araujo, E. A., Freitas, J. C. O. \& Braga, G. S. (2020). Efeito da goma xantana e da bentonita no desempenho de um fluido de perfuração base microemulsão. Holos, ano 36, v.2, e7358.

Souza, F. M., Soares, J. M. D., Oliveira, H. P., Rigoli, I. C., Luporini, S. (2020). Rheological assessment of the interaction between hydrophobic nanoclay and xanthan gum in saline environment, for application in drilling nanofluid. Research, Society and Development, v. 9, n. 7, 1-45, e789974669.

Souza, D. R. Q. A., Ferreira, G. F. D., Lobato, A. K. C. L., Silva, A. C. M. \& Santos, L. C. L. (2016). Influência do cotensoativo em sistemas microemulsionados. Revista Eletrônica de Petróleo e Gás, 4, 2, 33-42.

Souza, D. R. Q. A.; Oliveira, L. A.; Ferreira, G. F. D.; Lobato, A. K. C. L. \& Santos, L. C. L. (2015). Avaliação de tensoativos comerciais em sistemas microemulsionados na quebra de emulsão de petróleo In Anais...I Congresso Nacional de Engenharia de Petróleo, Gás Natural e Biocombustíveis, Campina Grande, PB, Brasil.

Souza, G. S., Luporini, S. \& Rigoli, I. C. (2017). Caracterização reológica de suspensões argilosas salinas com goma xantana para fluidos de perfuração de poços de petróleo. Revista Matéria, v.22, n.01, e11796.

Thomas, J. E. (2004). Fundamentos de engenharia de petróleo, 2 ed., Rio de Janeiro, Interciência, 2004.

Waldmann, A. T. A., Branco, M. A. \& Martins, A. (2007). Rheological characterization of polymer solutions for oil well drilling applications. In: Proceedings of the XI International Macromolecular Colloquium, Gramado, RS, Brasil. 